\title{
Measure of Departure From Local Symmetry for Square Contingency Tables
}

\author{
Yusuke Saigusa $^{1}$, Mitsuhiro Takami ${ }^{2}$, Aki Ishii ${ }^{2} \&$ Sadao Tomizawa $^{2}$ \\ ${ }^{1}$ Department of Biostatistics, Yokohama City University School of Medicine, Yokohama, Kanagawa 236-0004, Japan \\ ${ }^{2}$ Department of Information Sciences, Faculty of Science and Technology, Tokyo University of Science, Noda, Chiba \\ 278-8510, Japan
}

Correspondence: Yusuke Saigusa, Department of Biostatistics, Yokohama City University School of Medicine, Yokohama, Kanagawa 236-0004, Japan. E-mail: saigusay@yokohama-cu.ac.jp

Received: October 29, 2018 Accepted: November 23, 2018 Online Published: February 11, 2019

doi:10.5539/ijsp.v8n2p140

URL: https://doi.org/10.5539/ijsp.v8n2p140

\begin{abstract}
For square contingency tables, this paper considers the local symmetry model which indicates that there is a symmetric structure of probabilities for only one of pairs of symmetric cells. Also it proposes the measure to express the degree of departure from the local symmetry model. The measure is expressed as the weighted harmonic mean of the diversity index including the Shannon entropy. Examples are given.
\end{abstract}

Keywords: diversity index, local symmetry, measure, Shannon entropy, weighted harmonic mean

\section{Introduction}

For an $r \times r$ contingency table with the same row and column classifications, let $p_{i j}$ denote the probability that an observation will fall in the $i$ th row and $j$ th column of the table $(i=1, \ldots, r ; j=1, \ldots, r)$. The symmetry (S) model is defined by

$$
p_{i j}=\psi_{i j} \quad(i=1, \ldots, r ; j=1, \ldots, r),
$$

where $\psi_{i j}=\psi_{j i}$ for $i \neq j$ (Bowker, 1948; Bishop, Fienberg and Holland, 1975, p.282). For analyzing the data, when the $\mathrm{S}$ model fits the data poorly, we may be interested in measuring the degree of departure from the $\mathrm{S}$ model. Assume that $p_{i j}+p_{j i} \neq 0$ for $i \neq j$. Let $p_{i j}^{*}=p_{i j} / \delta$ and $p_{i j}^{c}=p_{i j} /\left(p_{i j}+p_{j i}\right)$ for $i \neq j$ with $\delta=\sum \sum_{i \neq j} p_{i j}$. Tomizawa (1994), and Tomizawa, Seo and Yamamoto (1998) proposed the measure to express the degree of departure from the $\mathrm{S}$ model as follows:

$$
\Phi_{S}^{(\lambda)}=\sum_{i=1}^{r-1} \sum_{j=i+1}^{r}\left(p_{i j}^{*}+p_{j i}^{*}\right) \Phi_{i j}^{(\lambda)} \quad \text { for } \lambda>-1,
$$

where

$$
\begin{gathered}
\Phi_{i j}^{(\lambda)}=1-\frac{\lambda 2^{\lambda}}{2^{\lambda}-1} H_{i j}^{(\lambda)}, \\
H_{i j}^{(\lambda)}=\left\{\begin{array}{lc}
\frac{1}{\lambda}\left(1-\left(p_{i j}^{c}\right)^{\lambda+1}-\left(p_{j i}^{c}\right)^{\lambda+1}\right) & \text { for } \lambda \neq 0, \\
-p_{i j}^{c} \log p_{i j}^{c}-p_{j i}^{c} \log p_{j i}^{c} & \text { for } \lambda=0,
\end{array}\right.
\end{gathered}
$$

and the value at $\lambda=0$ is taken to be the limit as $\lambda \rightarrow 0$, where $\lambda$ is a real-valued parameter which is chosen by user. Note that $H_{i j}^{(\lambda)}$ is the Patil and Tallies (1982) diversity index of degree $\lambda$ including the Shannon entropy when $\lambda=0$. We point out that $\Phi_{S}^{(\lambda)}$ is expressed as the weighted arithmetic mean of $\left\{\Phi_{i j}^{(\lambda)}\right\}$, i.e., the diversity index $\left\{H_{i j}^{(\lambda)}\right\}$. The measure $\Phi_{S}^{(\lambda)}$ lies between 0 and 1 , and $\Phi_{S}^{(\lambda)}=0$ if and only if the $S$ model holds. The measure $\Phi_{S}^{(\lambda)}$ is useful for measuring the degree of departure from the $\mathrm{S}$ model.

Saigusa, Tahata and Tomizawa (2016) proposed the measure $\Phi_{P S}^{(\lambda)}$, which is expressed as the weighted geometric mean of $\left\{\Phi_{i j}^{(\lambda)}\right\}$ as follows: 


$$
\Phi_{P S}^{(\lambda)}=\prod_{i=1}^{r-1} \prod_{j=i+1}^{r}\left[\Phi_{i j}^{(\lambda)}\right]^{\left(p_{i j}^{*}+p_{j i}^{*}\right)} \text { for } \lambda>-1
$$

The measure $\Phi_{P S}^{(\lambda)}$ lies between 0 and 1, and $\Phi_{P S}^{(\lambda)}=0$ if and only if the partial symmetry (PS) model holds, i.e.,

$$
p_{i j}=\psi_{i j} \quad(i=1, \ldots, r ; j=1, \ldots, r)
$$

where $\psi_{s t}=\psi_{t s}$ for at least one $(s, t)$ with $s \neq t$ (see Saigusa et al., 2016). The measure $\Phi_{P S}^{(\lambda)}$ is useful for measuring the degree of departure from the PS model.

Now we consider the new model expressed by

$$
p_{i j}=\psi_{i j} \quad(i=1, \ldots, r ; j=1, \ldots, r),
$$

where $\psi_{a b}=\psi_{b a}$ for only one $(a, b)$ with $a \neq b$. We shall refer to this model as the local symmetry (LS) model. Note that the $\mathrm{S}$ model implies the PS model and the PS model implies the LS model. We are also interested in measuring the degree of departure from the LS model.

The purpose of this paper is to propose the measure which is expressed as the weighted harmonic mean of the diversity index, instead of arithmetic and geometric means. The proposed measure is useful for measuring the degree of departure from the LS model. Section 2 proposes such a new measure. Section 3 gives the approximate confidence interval for the proposed measure. Section 4 shows examples. Sections 5 and 6 give the discussion and concluding remarks.

\section{Measure}

Assume that $p_{i j}+p_{j i} \neq 0$ for $i<j$, and $p_{k l}-p_{l k} \neq 0$ for any $k<l$ except $(k, l)=(a, b)$ with only one $(a, b), a<b$. Consider the measure defined by

$$
\Phi_{L S}^{(\lambda)}=\frac{1}{\sum_{i=1}^{r-1} \sum_{j=i+1}^{r} \frac{p_{i j}^{*}+p_{j i}^{*}}{\Phi_{i j}^{(\lambda)}}}=\frac{\prod_{s=1}^{r-1} \prod_{t=s+1}^{r} \Phi_{s t}^{(\lambda)}}{\sum_{i=1}^{r-1} \sum_{j=i+1}^{r}\left[\left(p_{i j}^{*}+p_{j i}^{*}\right) \prod_{s=1}^{r-1} \prod_{\substack{t=s+1 \\(s, t) \neq(i, j)}}^{r} \Phi_{s t}^{(\lambda)}\right]} \text { for } \lambda>-1
$$

where $\Phi_{s t}^{(\lambda)}$ is defined by (1). The measure $\Phi_{L S}^{(\lambda)}$ is expressed as the weighted harmonic mean of $\left\{\Phi_{i j}^{(\lambda)}\right\}$. For any $\lambda(>-1)$, we see

(i) $\Phi_{L S}^{(\lambda)}$ must lie between 0 and 1 ,

(ii) $\Phi_{L S}^{(\lambda)}=0$ if and only if the LS model holds,

(iii) $\Phi_{L S}^{(\lambda)}=1$ if and only if the degree of departure from LS is the largest in the sense that $p_{i j}^{c}=1\left(\right.$ then $\left.p_{j i}^{c}=0\right)$ or $p_{j i}^{c}=1\left(\right.$ then $\left.p_{i j}^{c}=0\right)$ for all $(i, j), i \neq j$.

The measure $\Phi_{L S}^{(\lambda)}$ is useful for measuring the degree of departure from the LS model, and the measure $\Phi_{S}^{(\lambda)}\left(\Phi_{P S}^{(\lambda)}\right)$ cannot be used to express the degree of departure from the LS model. We note that $\Phi_{L S}^{(\lambda)} \leq \Phi_{P S}^{(\lambda)} \leq \Phi_{S}^{(\lambda)}$.

\section{Approximate Confidence Interval of Measure}

Let $n_{i j}$ denote the observed frequency in the $(i, j)$ th cell of the square table $(i=1, \ldots, r ; j=1, \ldots, r)$. The sample version of $\Phi_{L S}^{(\lambda)}$, i.e. $\hat{\Phi}_{L S}^{(\lambda)}$, is given by $\Phi_{L S}^{(\lambda)}$ with $\left\{p_{i j}\right\}$ replaced by $\left\{\hat{p}_{i j}\right\}$, where $\hat{p}_{i j}=n_{i j} / n$ and $n=\sum \sum n_{i j}$. Assuming that a multinomial distribution applies to the $r \times r$ table, we provide an approximate standard error and large-sample confidence interval for the measure $\Phi_{L S}^{(\lambda)}$. We shall obtain the following theorem using the delta method (Agresti, 2013, p.587),

Theorem 1. $\sqrt{n}\left(\hat{\Phi}_{L S}^{(\lambda)}-\Phi_{L S}^{(\lambda)}\right)$ asymptotically $(n \rightarrow \infty)$ has a normal distribution with mean zero and variance $\sigma^{2}\left[\Phi_{L S}^{(\lambda)}\right]$, 
where

$$
\sigma^{2}\left[\Phi_{L S}^{(\lambda)}\right]=\sum_{i=1}^{r} \sum_{\substack{j=1 \\ j \neq i}}^{r} p_{i j}\left(\xi_{i j}^{(\lambda)}\right)^{2}-\left(\sum_{i=1}^{r} \sum_{\substack{j=1 \\ j \neq i}}^{r} p_{i j} \omega_{i j}^{(\lambda)}\right)^{2} \text { for } \lambda>-1,
$$

with

$$
\begin{aligned}
& \xi_{i j}^{(\lambda)}= \begin{cases}\frac{\left(\Phi_{L S}^{(\lambda)}\right)^{2}}{\delta}\left(\frac{1}{\Phi_{L S}^{(\lambda)}}-\frac{1}{\Phi_{i j}^{(\lambda)}}+\frac{2^{\lambda}(\lambda+1) p_{j i}^{c}}{\left(2^{\lambda}-1\right)\left(\Phi_{i j}^{(\lambda)}\right)^{2}}\left(\left(p_{i j}^{c}\right)^{\lambda}-\left(p_{j i}^{c}\right)^{\lambda}\right)\right) & \text { for } \lambda \neq 0, \\
\frac{\left(\Phi_{L S}^{(0)}\right)^{2}}{\delta}\left(\frac{1}{\Phi_{L S}^{(0)}}-\frac{1}{\Phi_{i j}^{(0)}}+\frac{p_{j i}^{c}}{(\log 2)\left(\Phi_{i j}^{(0)}\right)^{2}}\left(\log p_{i j}^{c}-\log p_{j i}^{c}\right)\right) & \text { for } \lambda=0,\end{cases} \\
& \omega_{i j}^{(\lambda)}= \begin{cases}\frac{\Phi_{L S}^{(\lambda)}}{\delta}\left(1-\frac{\Phi_{L S}^{(\lambda)}}{\Phi_{i j}^{(\lambda)}}\right) & \text { for } \lambda \neq 0, \\
\frac{\Phi_{L S}^{(0)}}{\delta}\left(1-\frac{\Phi_{L S}^{(0)}}{\Phi_{i j}^{(0)}}\right) & \text { for } \lambda=0 .\end{cases}
\end{aligned}
$$

This asymptotic distribution is not applicable when $\Phi_{L S}^{(\lambda)}=0$ and $\Phi_{L S}^{(\lambda)}=1$ because $\sigma^{2}\left[\Phi_{L S}^{(\lambda)}\right]=0$ when $\Phi_{L S}^{(\lambda)}=0$ and 1 . Let $\hat{\sigma}^{2}\left[\Phi_{L S}^{(\lambda)}\right]$ denote $\sigma^{2}\left[\Phi_{L S}^{(\lambda)}\right]$ with $\left\{p_{i j}\right\}$ replaced by $\left\{\hat{p}_{i j}\right\}$. Then $\hat{\sigma}\left[\Phi_{L S}^{(\lambda)}\right] / \sqrt{n}$ is an estimated approximate standard error for $\hat{\Phi}_{L S}^{(\lambda)}$, and $\hat{\Phi}_{L S}^{(\lambda)} \pm z_{\alpha / 2} \hat{\sigma}\left[\Phi_{L S}^{(\lambda)}\right] / \sqrt{n}$ is approximate $100(1-\alpha)$ percent confidence interval for $\Phi_{L S}^{(\lambda)}$, where $z_{\alpha / 2}$ is the upper $\alpha / 2$ th quantile of the standard normal distribution.

\section{Examples}

Consider the data in Table 1 taken from Tomizawa, Miyamoto and Iwamoto (2006). These are constructed from the data of decayed teeth of 363 women aged 18-39, for the patients visiting a dental clinic in Sapporo City, Japan, from 2001 to 2005. Table 1a is classified by the numbers of decayed teeth in the left side of the mouth of a patient and those in the right side. Note that each of these patients has at least one decayed tooth. Table $1 \mathrm{~b}$ is reclassified by the numbers of decayed teeth in the lower side of the mouth of a patient and those in the upper side.

Table 1. Decayed teeth data of 363 women aged 18-39, for patients visiting a dental clinic in Sapporo City, Japan, from 2001 to 2005 (Tomizawa et al., 2006)

(a) For the left and right decayed teeth

\begin{tabular}{ccccc}
\hline & \multicolumn{3}{l}{$\begin{array}{c}\text { Right (numbers } \\
\text { of decayed teeth) }\end{array}$} \\
\cline { 2 - 4 } Left (numbers of & $0-4$ & $5-8$ & $9+$ & Total \\
decayed teeth) & $(1)$ & $(2)$ & $(3)$ & \\
\hline $0-4 \quad(1)$ & 103 & 45 & 1 & 149 \\
$5-8 \quad(2)$ & 35 & 84 & 33 & 152 \\
$9+\quad(3)$ & 3 & 17 & 42 & 62 \\
\hline Total & 141 & 146 & 76 & 363 \\
\hline
\end{tabular}

(b) For the lower and upper decayed teeth

\begin{tabular}{ccccc}
\hline & \multicolumn{3}{l}{ Upper (numbers } \\
& of decayed teeth) & \\
\cline { 2 - 4 } Lower (numbers of & $0-4$ & $5-8$ & $9+$ & Total \\
decayed teeth) & $(1)$ & $(2)$ & $(3)$ & \\
\hline $0-4 \quad(1)$ & 97 & 62 & 15 & 174 \\
$5-8(2)$ & 20 & 63 & 75 & 158 \\
$9+\quad(3)$ & 2 & 6 & 23 & 31 \\
\hline Total & 119 & 131 & 113 & 363 \\
\hline
\end{tabular}


Table 2. The estimates of $\Phi_{L S}^{(\lambda)}$, estimated approximate standard errors of $\hat{\Phi}_{L S}^{(\lambda)}$ and approximate $95 \%$ confidence intervals of $\Phi_{L S}^{(\lambda)}$, applied to each of Tables $1 \mathrm{a}$ and $1 \mathrm{~b}$

(a) For Table 1a

\begin{tabular}{lccc}
\hline$\lambda$ & $\begin{array}{c}\text { Estimated } \\
\text { measure } \hat{\Phi}_{L S}^{(\lambda)}\end{array}$ & $\begin{array}{c}\text { Standard } \\
\text { error }\end{array}$ & $\begin{array}{c}\text { Confidence } \\
\text { interval }\end{array}$ \\
\hline-0.5 & 0.010 & 0.017 & $(-0.022,0.043)$ \\
0 & 0.017 & 0.028 & $(-0.038,0.072)$ \\
0.5 & 0.022 & 0.035 & $(-0.047,0.090)$ \\
1 & 0.024 & 0.039 & $(-0.052,0.099)$ \\
1.5 & 0.024 & 0.039 & $(-0.053,0.102)$ \\
2 & 0.024 & 0.039 & $(-0.052,0.099)$ \\
2.5 & 0.022 & 0.036 & $(-0.049,0.094)$ \\
3 & 0.021 & 0.033 & $(-0.045,0.086)$ \\
\hline
\end{tabular}

(b) For Table 1b

\begin{tabular}{lccc}
\hline$\lambda$ & $\begin{array}{c}\text { Estimated } \\
\text { measure } \hat{\Phi}_{L S}^{(\lambda)}\end{array}$ & $\begin{array}{c}\text { Standard } \\
\text { error }\end{array}$ & $\begin{array}{c}\text { Confidence } \\
\text { interval }\end{array}$ \\
\hline-0.5 & 0.198 & 0.061 & $(0.078,0.318)$ \\
0 & 0.311 & 0.088 & $(0.137,0.484)$ \\
0.5 & 0.370 & 0.100 & $(0.175,0.566)$ \\
1 & 0.397 & 0.104 & $(0.194,0.601)$ \\
1.5 & 0.404 & 0.104 & $(0.199,0.608)$ \\
2 & 0.397 & 0.104 & $(0.194,0.601)$ \\
2.5 & 0.382 & 0.102 & $(0.182,0.583)$ \\
3 & 0.363 & 0.101 & $(0.165,0.560)$ \\
\hline
\end{tabular}

Table 2 gives the estimated values of measure $\Phi_{L S}^{(\lambda)}$ applied to each of Tables 1a and 1b. It also gives the estimated approximate standard errors and the approximate $95 \%$ confidence intervals of the measure. From Table 2 , for any $\lambda$, the confidence interval of $\Phi_{L S}^{(\lambda)}$ applied to the data in Table 2a includes 0. So there may be a structure of LS in Table 2a. On the other hand, for any $\lambda$, the confidence interval of $\Phi_{L S}^{(\lambda)}$ applied to the data in Table $2 \mathrm{~b}$ does not include 0 . So there would not be a structure of LS in Table $2 b$.

We shall compare the degrees of departure from LS for Tables $2 \mathrm{a}$ and $2 \mathrm{~b}$ using $\Phi_{L S}^{(\lambda)}$. Comparing the confidence intervals of $\Phi_{L S}^{(\lambda)}$ for any $\lambda$, it is inferred that the degree of departure from LS for Table $2 \mathrm{~b}$ is larger than that for Table $2 \mathrm{a}$.

\section{Discussion}

Consider the $4 \times 4$ artificial cell probability tables given in Table 3 . There is a structure of LS in Table $3 \mathrm{a}$ with $p_{12}=p_{21}=$ 0.02. For each of Tables $3 \mathrm{~b}, 3 \mathrm{c}, 3 \mathrm{~d}$ and $3 \mathrm{e}$, the value of $p_{k l}$ for any $k \neq l$ except $(k, l)=(2,1)$ equals the value of $p_{k l}$ in Table 3a. Also the ratio $p_{21} / p_{12}$ is 1.0 for Table 3a, 2.0 for Table 3b, 3.0 for Table $3 \mathrm{c}, 4.0$ for Table $3 \mathrm{~d}$, and 5.0 for Table 3e. Also Table $3 \mathrm{f}$ shows that the probabilities in upper right triangle cells are zeros. From Table 4, we see that (1) the value of $\Phi_{L S}^{(\lambda)}$ for Table 3a equals zero, (2) for any fixed $\lambda$, the value of $\Phi_{L S}^{(\lambda)}$ increases as the ratio $p_{21} / p_{12}$ increases, and (3) the value of $\Phi_{L S}^{(\lambda)}$ for Table 3 f equals 1 . Therefore the measure $\Phi_{L S}^{(\lambda)}$ would be natural to describe the degree of departure from the LS model.

\section{Concluding Remarks}

We have considered the LS model (in Section 1). The LS model indicates the structure of $\left\{p_{i j}\right\}$ which has weaker restrictions than those for the PS and S models. Also, we have proposed the measure $\Phi_{L S}^{(\lambda)}$ for describing the degree of departure from the LS model. The $\Phi_{L S}^{(\lambda)}$ is useful to see how far cell probabilities are distant from those with the LS structure, as the $\Phi_{S}^{(\lambda)}\left(\Phi_{P S}^{(\lambda)}\right)$ is useful to see how far cell probabilities are distant from those with the S (PS) structure.

We point out that the measures $\Phi_{S}^{(\lambda)}$ and $\Phi_{P S}^{(\lambda)}$ cannot express the degree of departure from the LS model (as described in Section 2). The measure $\Phi_{L S}^{(\lambda)}$ is useful for comparing the degrees of departure from the LS model in several square tables.

We note that the measure $\Phi_{L S}^{(\lambda)}$ (also $\Phi_{S}^{(\lambda)}$ and $\Phi_{P S}^{(\lambda)}$ ) is invariant under arbitrary similar permutations of row and column categories. Therefore it is possible to apply these measures for analyzing data that are on a nominal or ordinal scale. 
Table 3. Artificial cell probability tables

(a)

\begin{tabular}{llll}
\hline 0.184 & 0.020 & 0.019 & 0.009 \\
0.020 & 0.184 & 0.001 & 0.004 \\
0.076 & 0.004 & 0.184 & 0.012 \\
0.036 & 0.016 & 0.048 & 0.183 \\
\hline
\end{tabular}

(c)

\begin{tabular}{llll}
\hline 0.173 & 0.020 & 0.019 & 0.009 \\
0.060 & 0.174 & 0.001 & 0.004 \\
0.076 & 0.004 & 0.174 & 0.012 \\
0.036 & 0.016 & 0.048 & 0.174 \\
\hline
\end{tabular}

(e)

\begin{tabular}{llll}
\hline 0.163 & 0.020 & 0.019 & 0.009 \\
0.100 & 0.164 & 0.001 & 0.004 \\
0.076 & 0.004 & 0.164 & 0.012 \\
0.036 & 0.016 & 0.048 & 0.164 \\
\hline
\end{tabular}

(b)

\begin{tabular}{llll}
\hline 0.178 & 0.020 & 0.019 & 0.009 \\
0.040 & 0.179 & 0.001 & 0.004 \\
0.076 & 0.004 & 0.179 & 0.012 \\
0.036 & 0.016 & 0.048 & 0.179 \\
\hline
\end{tabular}

(d)

\begin{tabular}{llll}
\hline 0.168 & 0.020 & 0.019 & 0.009 \\
0.080 & 0.169 & 0.001 & 0.004 \\
0.076 & 0.004 & 0.169 & 0.012 \\
0.036 & 0.016 & 0.048 & 0.169 \\
\hline
\end{tabular}

(f)

\begin{tabular}{llll}
\hline 0.215 & 0 & 0 & 0 \\
0.079 & 0.215 & 0 & 0 \\
0.013 & 0.004 & 0.215 & 0 \\
0.021 & 0.018 & 0.005 & 0.215 \\
\hline
\end{tabular}

Table 4. Values of $\Phi_{L S}^{(\lambda)}$ applied to each of Tables 3a-f

\begin{tabular}{cccc}
\hline Applied & \multicolumn{3}{c}{$\lambda$} \\
\cline { 2 - 4 } tables & 0 & 0.5 & 1.5 \\
\hline Table 3a & 0.000 & 0.000 & 0.000 \\
Table 3b & 0.185 & 0.225 & 0.250 \\
Table 3c & 0.247 & 0.299 & 0.329 \\
Table 3d & 0.278 & 0.334 & 0.366 \\
Table 3e & 0.299 & 0.359 & 0.392 \\
Table 3f & 1.000 & 1.000 & 1.000 \\
\hline
\end{tabular}

\section{Acknowledgements}

The authors would like to thank editor and referees for their helpful comments.

\section{References}

Agresti, A. (2013). Categorical Data Analysis, 3rd Edition. John Wiley and Sons, Hoboken, New Jersey. https://doi.org/10.1111/biom.12128

Bishop, Y. M. M., Fienberg S. E., \& Holland, P. W. (1975). Discrete Multivariate Analysis: Theory and Practice. MIT Press, Cambridge. https://doi.org/10.1007/978-0-387-72806-3

Bowker, A. H. (1948). A test for symmetry in contingency tables. Journal of the American Statistical Association, 43, 572-574. https://doi.org/10.1080/01621459.1948.10483284

Patil, G. P., \& Taillie, C. (1982). Diversity as a concept and its measurement. Journal of the American Statistical Association, 77, 548-561. https://doi.org/10.1080/01621459.1982.10477845

Saigusa,Y., Tahata, K., \& Tomizawa, S. (2016). Measure of departure from partial symmetry for square contingency tables. Journal of Mathematics and Statistics, 12, 152-156. https://doi.org/10.3844/jmssp.2016.152.156

Tomizawa, S. (1994). Two kinds of measures of departure from symmetry in square contingency tables having nominal categories. Statistica Sinica, 4, 325-334.

Tomizawa, S., Seo, T., \& Yamamoto, H. (1998). Power-divergence-type measure of departure from symmetry for square 
contingency tables that have nominal categories. Journal of Applied Statistics, 25, 387-398. https://doi.org/10.1080/02664769823115

Tomizawa, S., Miyamoto, N., \& Iwamoto, M. (2006). Linear column-parameter symmetry model for square contingency tables: application to decayed teeth data. Biometrical Letters, 43, 91-98.

\section{Copyrights}

Copyright for this article is retained by the author(s), with first publication rights granted to the journal.

This is an open-access article distributed under the terms and conditions of the Creative Commons Attribution license (http://creativecommons.org/licenses/by/4.0/). 